in vivo $35: 3627-3631(2021)$

doi:10.21873/invivo.12669

\title{
Distal Pancreatectomy With Celiac Axis Resection for Locally Advanced Pancreatic Body Cancer - A Case Report and Literature Review
}

\author{
NICOLAE BACALBASA ${ }^{1,2}$, IRINA BALESCU ${ }^{3}$, MIHAI DIMITRIU ${ }^{2,4}$, \\ CRISTIAN BALALAU ${ }^{5,6}$, FLORENTINA FURTUNESCU ${ }^{7}$, FLORENTINA GHERGHICEANU ${ }^{8}$, \\ DANIEL RADAVOI ${ }^{9,10}$, CAMELIA DIACONU ${ }^{11,12}$, OVIDIU STIRU ${ }^{13,14}$, CORNEL SAVU ${ }^{15,16}$, \\ VLADISLAV BRASOVEANU ${ }^{1}$, CLAUDIA STOICA ${ }^{17,18}$ and IOAN CORDOS ${ }^{15,16}$ \\ ${ }^{1}$ Department of Visceral Surgery, Center of Excellence in Translational \\ Medicine Fundeni Clinical Institute, Bucharest, Romania; \\ ${ }^{2}$ Department of Obstetrics and Gynecology, Carol Davila University of Medicine and Pharmacy, Bucharest, Romania; \\ ${ }^{3}$ Department of Surgery Ponderas Academic Hospital, Bucharest, Romania; \\ ${ }^{4}$ Department of Obstetrics and Gynecology, St. Panteleimon Emergency Hospital, Bucharest, Romania; \\ ${ }^{5}$ Department of Surgery, Carol Davila University of Medicine and Pharmacy, Bucharest, Romania; \\ ${ }^{6}$ Department of Surgery, St. Panteleimon Emergency Hospital, Bucharest, Romania; \\ ${ }^{7}$ Department of Public Health and Management, Carol Davila University \\ of Medicine and Pharmacy, Bucharest, Romania; \\ ${ }^{8}$ Department of Marketing and Medical Technology, Carol Davila \\ University of Medicine and Pharmacy, Bucharest, Romania; \\ ${ }^{9}$ Department of Urology, Prof. Dr. Th. Burghele Clinical Hospital, Bucharest, Romania; \\ ${ }^{10}$ Department of Urology, Carol Davila University of Medicine and Pharmacy, Bucharest, Romania; \\ ${ }^{11}$ Department of Internal Medicine, Carol Davila University of Medicine and Pharmacy, Bucharest, Romania; \\ ${ }^{12}$ Department of Internal Medicine, Clinical Emergency Hospital of Bucharest, Bucharest, Romania; \\ ${ }^{13}$ Prof. Dr. C.C. Iliescu Emergency Institute for Cardiovascular Diseases, Bucharest, Romania; \\ ${ }^{14}$ Department of Cardio-Thoracic Pathology, Carol Davila University of Medicine and Pharmacy, Bucharest, Romania; \\ ${ }^{15}$ Department of Thoracic Surgery, Marius Nasta National Institute of Pneumology, Bucharest, Romania; \\ ${ }^{16}$ Department of Thoracic Surgery, Carol Davila University of Medicine and Pharmacy, Bucharest, Romania; \\ ${ }^{17}$ Department of Surgery, Ilfov County Hospital, Bucharest, Romania; \\ ${ }^{18}$ Department of Anatomy, Carol Davila University of Medicine and Pharmacy, Bucharest, Romania
}

\begin{abstract}
Background: Locally advanced pancreatic cancer invading the surrounding vascular structures has long been considered as unresectable and, therefore, patients were usually submitted to palliative chemotherapy. Case Report: We present the case of a 44-year-old male investigated for weight loss and abdominal pain and diagnosed with a locally
\end{abstract}

This article is freely accessible online.

Correspondence to: Nicolae Bacalbasa, Carol Davila University of Medicine and Pharmacy, 37 Dionisie Lupu Street, 020021 Bucharest, Romania. Tel: +40 723540426, e-mail: nicolae_bacalbasa@yahoo.ro

Key Words: Pancreatic cancer, locally advanced disease, celiac axis, resection. advanced pancreatic tumor invading the celiac axis. An endoscopic ultrasound was performed and a biopsy was retrieved demonstrating the presence of a moderately differentiated pancreatic adenocarcinoma. After discussing with the patient the risks and the benefits of performing an extended surgical procedure, the patient consented to distal pancreatectomy en bloc with celiac axis resection. Postoperatively, the patient was submitted to low-molecularweight heparin therapy for 3 weeks followed by oral anticoagulant for 2 months. Histopathological studies confirmed the presence of a moderately differentiated pancreatic adenocarcinoma invading the celiac axis and described negative resection margins. Conclusion: Although celiac axis invasion has been considered for a long period of time as a sign of unresectable disease due to the high rates of perioperative complications, it seems that in selected 
cases, surgery can be safely performed with curative intent, especially if negative resection margins are achieved.

Locally advanced pancreatic cancer has long been considered as an unresectable disease and therefore patients diagnosed with this pathology are referred for palliative oncological treatment, with an overall lifespan of less than 1 year $(1,2)$. For some, this aspect was explained by the fact that in cases presenting arterial invasion (and therefore considered as having a locally advanced pancreatic lesion), perineural invasion is also present and the risk of having distant micrometastases at the time of resection is higher (2); therefore, a clear distinction was made between cases presenting arterial and venous invasion. In this respect, cases presenting venous invasion are considered as borderline resectable and such patients are considered for surgery, while those presenting arterial invasion (alone or, most often, associated with venous invasion) are considered as having locally advanced disease and are referred for medical oncological treatment (3-6). However, with improvement in surgical techniques as well as the perioperative management, and with new therapeutic agents being introduced as part of systemic therapy, in such cases the overall survival has also improved and arterial resection can be reconsidered in selected cases (7-9). This method is particularly efficient in cases in which negative resection margins are achieved. Although the most commonly encountered cases in which arterial resection has been reported refer to cancer of the pancreatic head with invasion of the superior mesenteric/hepatic artery/celiac trunk, the method has also been reported for patients with pancreatic body tumors with celiac axis invasion (10-14). Our aim is to report the case of patient diagnosed with pancreatic body adenocarcinoma with celiac axis invasion who was successfully submitted to resection.

\section{Case Report}

After obtaining the approval of the Ethics Committee (no. 27/2021) data of the patient was retrieved and presented in the current report.

A 44-year-old previously healthy patient was investigated for diffuse epigastric pain associated with weight loss of 12 $\mathrm{kg}$ in the previous 2 months in January 2020; the patient denied having other signs and symptoms such as vomiting or modification of the appearance of stool/urine. The patient was submitted to an abdominal ultrasound which revealed the presence of a $43 \times 38 \times 35 \mathrm{~mm}$ tumor of the pancreatic body developed in the close proximity to the celiac trunk. The patient was further submitted to computed tomography, which confirmed the information given by ultrasound and to an endoscopic ultrasound; during this process a biopsy was retrieved and demonstrated the presence of a moderately differentiated pancreatic adenocarcinoma. Endoscopic ultrasound confirmed the encasement of the celiac axis extended a length of approximately $0.5 \mathrm{~cm}$.

After discussing with the patient the possibilities of treatment, their benefits and risks, and after obtaining his informed consent, the patient was submitted to surgery with curative intent. Intraoperatively the presence of local but limited invasion of the celiac axis was confirmed. Therefore, distal pancreatectomy en bloc with splenectomy and celiac axis resection was performed; the celiac axis was sectioned at the origin and the common hepatic artery was clamped (Figure 1). The vascularization of the liver through the gastroduodenal artery was checked but intraoperative ultrasound failed to demonstrate the presence of an adequate hepatic inflow, therefore the common hepatic artery was reimplanted at the level of the abdominal aorta (Figure 2). At the end of the surgical procedure there was no sign of gastric ischemia and gastric resection was consequently considered as unnecessary.

There were no intraoperative incidents; the intraoperative blood loss was $650 \mathrm{ml}$, while the length of the operative procedure was 480 minutes; during the operative procedure, two units of red blood cells were administered. The postoperative evolution was uneventful, the patient being discharged on the $10^{\text {th }}$ postoperative day. Low-molecularweight heparin therapy was administered for the following 3 weeks and was followed by oral anticoagulant for 2 months. The histopathological studies confirmed the presence of a moderately differentiated pancreatic adenocarcinoma invading the celiac axis and described negative resection margins. Postoperatively, the patient was referred to the oncology service in order to be submitted to adjuvant chemotherapy. At the 1-year follow-up, the patient remained alive and free of any recurrent disease.

\section{Discussion}

Distal pancreatectomy en bloc with celiac axis resection was initially described by Appleby in 1950 for locally advanced gastric cancer and was associated at that time with total gastrectomy (15); the method was further implemented in locally advanced pancreatic cancer with celiac axis invasion with or without total gastrectomy in 1997-1998 (16-18). However, the method has been associated with increased rates of perioperative complications such as hepatic or gastric ischemia, pancreatic leaks or postoperative hemorrhage, and has been intensely discussed $(7,10,19,20)$.

As expected, the most fearful complications which should be taken into consideration when performing such surgical procedures are related to the risk of gastric and, in particular, hepatic ischemia; theoretically, after performing such a procedure, gastric ischemia is prevented by the collateral blood flow through the right gastric artery, right gastroepiploic artery and left phrenic artery, while the liver 


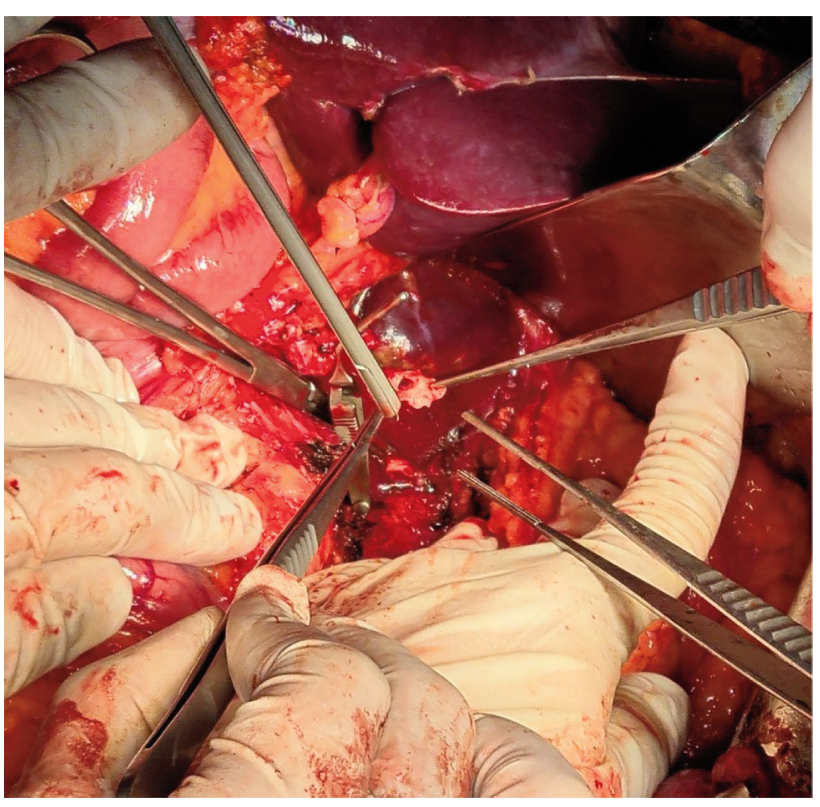

Figure 1. Intraoperative aspect after distal pancreatectomy en bloc with splenectomy and celiac axis resection. The common hepatic artery was clamped and the patency of liver vascularization through the gastroduodenal artery was checked.

is vascularized through the gastroduodenal artery and through the pancreatic head arcade. However, while in cases in which gastric ischemia is suspected the procedure might be completed by the association of total gastrectomy, in cases in which intraoperative ultrasound shows poor vascularization of the liver through the pancreatic head and gastroduodenal artery, more complicated surgical procedures might be needed. In this respect, certain authors take into consideration preoperative embolization of the common hepatic artery in order to increase the blood flow through the pancreatic arcade and to stimulate the development of collateral circulation or even arterial reconstruction or bypass $(19,20)$. Machado et al., in 2009, proposed performing an arterial bypass from the common hepatic artery to the left external iliac artery by placing a Dacron prosthesis; the method proved to be a successful one, the patient being alive and asymptomatic 13 months after surgery (19).

A very interesting study which aimed to investigate the benefits of celiac axis resection in locally advanced pancreatic cancer was published by Yamamoto et al. in 2012 (7); the study included 13 cases submitted to celiac axis resection and distal pancreatectomy for locally advanced pancreatic cancer, 58 cases submitted to surgery for the same stage of the disease but in which celiac axis resection was not needed, and 24 cases which proved to have unresectable lesions due to the local extent. The authors underlined the fact that patients included in the first group were submitted to

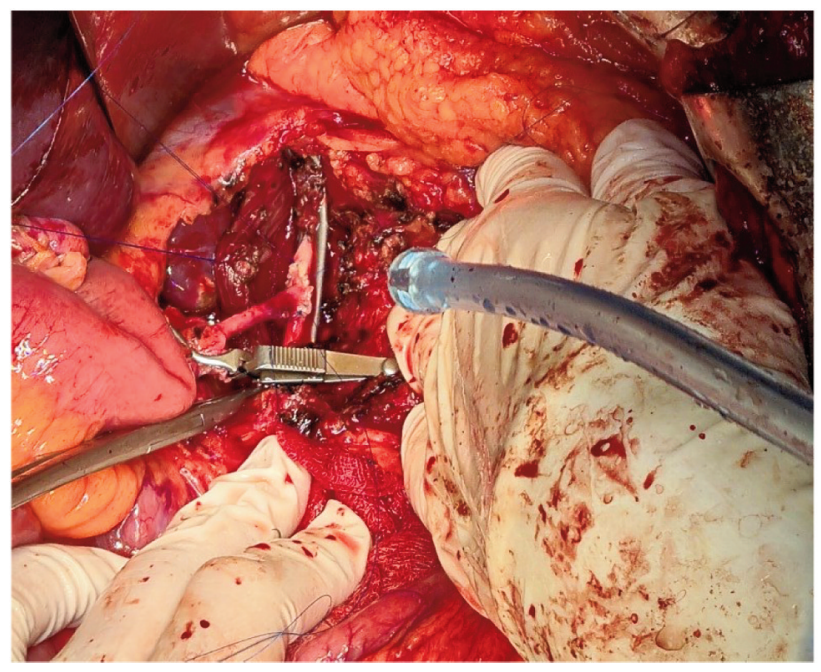

Figure 2. The final aspect after reimplantation of the common hepatic artery at the level of the abdominal aorta.

significantly longer surgical procedures, experienced greater amounts of blood loss and more often underwent portal vein resections or total gastrectomy in order to prevent gastric ischemia; the overall morbidity was significantly higher in the first group, whilst the rate of negative resection margins was significantly lower. However, long-term survival for patients included in the first two groups was significantly improved when compared to those of the third group. Therefore, the authors underlined the fact that in selected cases this surgical procedure is perfectly justified and might provide a significant survival benefit, although increased rates of perioperative morbidity are to be expected (7).

As can be seen from the above-mentioned study, a significant issue which should be taken into consideration when performing such an extended procedure is the risk of positive resection margins; in such cases, the overall survival might be significantly reduced and therefore this type of procedure becomes an unjustified one. In order to identify which cases might benefit most from such surgical procedures, a recent study conducted by Truty et al. (10) at the Mayo Clinic stratified celiac axis invasion using a threelevel classification. The study included cases diagnosed with locally advanced pancreatic cancer submitted to celiac axis resection between January 1995 and September 2019. Patients included in the first category presented celiac axis invasion with no further extension at the level of the proper hepatic artery/gastro-duodenal artery. These patients were further classified into three subcategories: the first included patients submitted to classic Appleby procedure - with adequate collateral circulation and no need for reperfusion, the second included cases in which proper hepatic vascularization could not be achieved after resection and 
therefore needed further hepatic artery reconstruction, while the third subgroup included cases in which gastrectomy was needed due to inadequate gastric perfusion after resection. Cases included in the second group were diagnosed with celiac axis invasion at the level of the celiac axis branches in association with the absence of a patent collateral circulation. Depending on the extent of disease at the level of the pancreatic head, these patients were divided into two subcategories: the first presented no extension at this level and therefore subtotal pancreatectomy was feasible, while those included in the second subcategory presented pancreatic head invasion and were submitted to total pancreatectomy en bloc with total gastrectomy and hepatic revascularization. Cases included in the third group presented tumors invading both the celiac axis and the superior mesenteric artery and were submitted to distal pancreatectomy en bloc with celiac axis and superior mesenteric artery resection, followed by hepatic and bowel revascularization, with or without total gastrectomy (the first subgroup) and subtotal/total pancreatectomy en bloc with total gastrectomy, hepatic and bowel revascularization (the two subcategories were established depending of the extent of the vascular invasion at the level of the celiac axis branches and of the patency of collateral circulation). After a median follow-up of 24.6 months, the recurrence rate was $44 \%$, while the median overall survival was 36.2 months. The authors underlined the fact that the long-term outcomes were significantly improved in cases submitted to neoadjuvant chemotherapy. Other predictors for improved long-term outcomes were represented by the lymph node status, as well as by the normalization of the serum level of CA19-9. When analyzing the influence of the type of anatomical invasion of the celiac axis, no significant difference in terms of survival was observed between the three categories of patients. However, this classification system seems to offer a better operative planning strategy for cases in which such extended surgical procedures are planned (10).

These data demonstrate once again that whenever a proper technical surgical reconstruction is performed, and negative resection margins are achieved, improved long-term outcomes should be expected. However, in order to give the patient the chance to benefit from such an extended procedure, a proper intraoperative evaluation of the extent of the disease and of the patency of the collateral circulation is mandatory; cases presenting an adequate collateral circulation can be submitted to less complex surgical procedures without association of complex vascular reconstruction. When it comes to the case that we presented, the intraoperative evaluation failed to demonstrate efficient vascularization through the collateral network and therefore the common hepatic artery was further implanted at the level of the abdominal aorta. The absence of signs of gastric necrosis gave us the opportunity to avoid completing the surgical procedure with a partial gastrectomy, whilst achieving negative resection margins by histopathological studies enabled us to consider that long-term survival is to be expected.

\section{Conclusion}

Although initially considered as a sign of unresectable disease, celiac axis invasion in cancer of the pancreatic body should not be considered as a formal contraindication for surgery. In certain cases presenting a good biological and clinical status, as well as limited local invasion, en bloc resection of the celiac trunk with distal pancreatectomy might be performed. However, after such extended procedures, severe complications such as liver ischemia might occur; whenever intraoperative ultrasound raises the suspicion of inadequate liver inflow, arterial reconstruction should be considered.

\section{Conflicts of Interest}

None declared.

\section{Authors' Contribution}

Conceptualization, N.B. and M.D.; methodology, C.B. and I.C.; formal analysis, F.F. and F.G.; investigation, C.D.; resources, O.S.; data curation, C.St.; writing-original draft preparation, I.B.; writing - review and editing, C.S.; visualization, D.R.; supervision, V.B, I.C. All Authors read and agreed to the submitted version of the article.

\section{References}

1 Sawaki A, Hoki N, Ito S, Matsumoto K, Mizuno N, Hara K, Takagi T, Kobayashi Y, Sawai Y, Kawai H, Tajika M, Nakamura $\mathrm{T}$ and Yamao K: Clinical impact of radiotherapy for locally advanced pancreatic cancer. J Gastroenterol 44(12): 1209-1214, 2009. PMID: 19705054. DOI: 10.1007/s00535-009-0116-9

2 Katz MH, Hwang R, Fleming JB and Evans DB: Tumor-nodemetastasis staging of pancreatic adenocarcinoma. CA Cancer J Clin 58(2): 111-125, 2008. PMID: 18272835. DOI: $10.3322 / \mathrm{CA}$. 2007.0012

3 Barreto SG and Kleeff J: Synchronous arterial resections in pancreatic cancer - still a matter of debate? Eur J Surg Oncol 47(2): 480-482, 2021. PMID: 32586727. DOI: 10.1016/j.ejso.2020.06.028

4 Małczak P, Sierżęga M, Stefura T, Kacprzyk A, Droś J, Skomarovska O, Krzysztofik M, Major P and Pędziwiatr M: Arterial resections in pancreatic cancer - Systematic review and meta-analysis. HPB (Oxford) 22(7): 961-968, 2020. PMID: 32360186. DOI: $10.1016 /$ j.hpb.2020.04.005

5 Haines M, Chua TC, Jamieson NB, Mittal A, Gill AJ and Samra JS: Pancreatoduodenectomy with arterial resection for locally advanced pancreatic cancer of the head: a systematic review. Pancreas 49(5): 621-628, 2020. PMID: 32433398. DOI: 10.1097/MPA.0000000000001551 
6 Butler JR, Ahmad SA, Katz MH, Cioffi JL and Zyromski NJ: A systematic review of the role of periadventitial dissection of the superior mesenteric artery in affecting margin status after pancreatoduodenectomy for pancreatic adenocarcinoma. HPB (Oxford) 18(4): 305-311, 2016. PMID: 27037198. DOI: 10.1016/j.hpb.2015.11.009

7 Yamamoto Y, Sakamoto Y, Ban D, Shimada K, Esaki M, Nara S and Kosuge T: Is celiac axis resection justified for T4 pancreatic body cancer? Surgery 151(1): 61-69, 2012. PMID: 22088810. DOI: $10.1016 /$ j.surg.2011.06.030

8 Ishii H, Furuse J, Boku N, Okusaka T, Ikeda M, Ohkawa S, Fukutomi A, Hamamoto Y, Nakamura K, Fukuda H and JCOG Gastrointestinal Oncology Study Group: Phase II study of gemcitabine chemotherapy alone for locally advanced pancreatic carcinoma: JCOG0506. Jpn J Clin Oncol 40(6): 573-579, 2010. PMID: 20185458. DOI: 10.1093/jjco/hyq011

9 Nakachi K, Furuse J, Kinoshita T, Kawashima M, Ishii H, Ikeda M, Mitsunaga S and Shimizu S: A phase II study of induction chemotherapy with gemcitabine plus S-1 followed by chemoradiotherapy for locally advanced pancreatic cancer. Cancer Chemother Pharmacol 66(3): 527-534, 2010. PMID: 19967537. DOI: $10.1007 / \mathrm{s} 00280-009-1193-4$

10 Truty MJ, Colglazier JJ, Mendes BC, Nagorney DM, Bower TC, Smoot RL, DeMartino RR, Cleary SP, Oderich GS and Kendrick ML: En bloc celiac axis resection for pancreatic cancer: Classification of anatomical variants based on tumor extent. J Am Coll Surg 231(1): 8-29, 2020. PMID: 32422348. DOI: 10.1016/j.jamcollsurg.2020.05.005

11 Brasoveanu V, Anghel C, Barbu I, Pautov M, Ionescu MI, Motthor M, Balescu I, Dima $\mathrm{S}$ and Bacalbasa $\mathrm{N}$ : Pancreatoduodenectomy en bloc with portal and superior mesenteric artery resection-a case report and literature review. Anticancer Res 35(3): 1613-1618, 2015. PMID: 25750318.

12 Braşoveanu V, Dumitraşcu T, Bacalbaşa N and Zamfir R: Splenic artery used for replaced common hepatic artery reconstruction during pancreatoduodenectomy - a case report. Chirurgia (Bucur) 104(4): 499-504, 2009. PMID: 19886062.

13 Bacalbasa N, Balescu I, Tanase A, Pautov M, Brezean I, Vilcu $\mathrm{M}$ and Brasoveanu V: Spleno-pancreatectomy en bloc with parcelar gastrectomy for splenic artery aneurysm - a case report and literature review. In Vivo 32(4): 915-919, 2018. PMID: 29936480. DOI: 10.21873/invivo.11329
14 Bacalbasa N, Balescu I, Tanase A, Brezean I, Vilcu M and Brasoveanu V: Successful resection of a non-functional paraganglioma with celiac trunk invasion followed by common hepatic artery reimplantation - a case report and literature review. In Vivo 32(4): 911-914, 2018. PMID: 29936479. DOI: 10.21873/invivo.11328

15 Appleby LH: The coeliac axis in the expansion of the operation for gastric carcinoma. Cancer 6(4): 704-707, 1953. PMID: 13059764. DOI: 10.1002/1097-0142(195307)6:4<704::aid-cncr 2820060410>3.0.co;2-p

16 Kimura W, Han I, Furukawa Y, Sunami E, Futakawa N, Inoue T, Shinkai H, Zhao B, Muto T, Makuuchi M and Komatsu H: Appleby operation for carcinoma of the body and tail of the pancreas. Hepatogastroenterology 44(14): 387-393, 1997. PMID: 9164507.

17 Mayumi T, Nimura Y, Kamiya J, Kondo S, Nagino M, Kanai M, Miyachi M, Hamaguchi $\mathrm{K}$ and Hayakawa N: Distal pancreatectomy with en bloc resection of the celiac artery for carcinoma of the body and tail of the pancreas. Int J Pancreatol 22(1): 15-21, 1997. PMID: 9387020. DOI: 10.1007/BF02803900

18 Ozaki H, Kinoshita T, Kosuge T, Yamamoto J, Shimada K, Inoue K, Koyama Y and Mukai K: An aggressive therapeutic approach to carcinoma of the body and tail of the pancreas. Cancer 77(11): 2240-2245, 1996. PMID: 8635090. DOI: 10.1002/(SICI)10970142(19960601)77:11<2240::AID-CNCR9>3.0.CO;2-T

19 Machado MA, Surjan RC, Nishinari K, Makdissi FF and Machado MC: Iliac-hepatic arterial bypass for compromised collateral flow during modified Appleby operation for advanced pancreatic cancer. Eur J Surg Oncol 35(10): 1124-1127, 2009. PMID: 19410414. DOI: 10.1016/j.ejso.2009.04.005

20 Hirai I, Kimura W, Kamiga M, Mizutani M, Takeshita A, Watanabe $\mathrm{T}$ and Fuse A: The significance of intraoperative Doppler ultrasonography in evaluating hepatic arterial flow when assessing the indications for the Appleby procedure for pancreatic body cancer. J Hepatobiliary Pancreat Surg 12(1): 5560, 2005. PMID: 15754101. DOI: 10.1007/s00534-004-0939-y

Received June 2, 2021

Revised July 29, 2021

Accepted August 26, 2021 